\title{
Recursive Robust Estimation and Control Without Commitment
}

\author{
Lars Peter Hansen* \\ Thomas J. Sargent ${ }^{\ddagger}$
}

May 2, 2006

\begin{abstract}
In a Markov decision problem with hidden state variables, a posterior distribution serves as a state variable and Bayes' law under an approximating model gives its law of motion. A decision maker expresses fear that his model is misspecified by surrounding it with a set of alternatives that are nearby when measured by their expected log likelihood ratios (entropies). Martingales represent alternative models. A decision maker constructs a sequence of robust decision rules by pretending that a sequence of minimizing players choose increments to a martingale and distortions to the prior over the hidden state. A risk sensitivity operator induces robustness to perturbations of the approximating model conditioned on the hidden state. Another risk sensitivity operator induces robustness to the prior distribution over the hidden state. We use these operators to extend the approach of Hansen and Sargent (1995) to problems that contain hidden states.
\end{abstract}

\section{Introduction}

This paper constructs robust decision rules for discounted dynamic programming problems that confront a decision maker with an incentive to evaluate alternative models and to learn about unknown parameters and other hidden state variables. ${ }^{1}$ Extending robust control theory to include these features allows us to approach unsolved problems in positive and normative economics, for example, computing asset evaluations of robust investors and designing robust monetary and fiscal policies. A criticism of our earlier robust control formulations with persistent fears of model misspecification (for example, Hansen and Sargent (1995), Hansen et al. (2006a), Hansen et al. (1999), Anderson et al. (2003)) is that they precluded learning by not allowing the decision maker to use new information to refine his

\footnotetext{
*University of Chicago. Email: l-hansen@uchicago.edu

${ }^{\dagger}$ New York University and Hoover Institution. Email: ts43@nyu.edu

${ }^{\ddagger}$ We thank Anastasios Karantounias, Ricardo Mayer, Grace Tsiang, and especially Tomasz Piskorski for helpful comments on earlier drafts of this paper. We thank In-Koo Cho for encouragement.

${ }^{1}$ For examples of such dynamic programming problems, see Jovanovic (1979), Jovanovic and Nyarko (1995, 1996), and Bergemann and Valimaki (1996).
} 
approximating model and diminish the set of plausible alternatives against which he seeks robustness. $^{2}$ This paper confronts that criticism by incorporating learning.

The Bayesian literature on model selection and learning has acquired prominent critics. Turning their backs on Bayes' law and the logic of backward induction, distinguished macroeconomists have recommended against using experimentation, learning, and model selection to guide decision making. ${ }^{3}$ These skeptics seem to believe that in any concrete application, the details of a model are bound to be subject to misspecifications that will render a recommendation to experiment that comes from pursuing the logic of Bayes and backward induction unacceptably fragile. ${ }^{4}$ Our methods show a decision maker how to experiment even though he distrusts both his models and the prior that he places over them.

We begin by assuming that, through some unspecified process, a decision maker has arrived at an approximating model that fits historical data well. Our notion of a model is broad enough to subsume the possibility that the model is actually a collection of alternative submodels, each of which can contain unknown parameters. More generally, we formulate the decision environment using a hidden state Markov model, and we index a family of probability models by conditioning on the hidden Markov state. Some of the components of the Markov states can be time invariant indicators of submodels and others can be parameters or hidden states associated with the submodels that vary over time. A conditional model is a probability specification conditioned on a hidden Markov state. Because the decision-maker fears that each member of his family of approximating conditional models is misspecified, he surrounds each of them with a set of unspecified alternative models whose expected log likelihood ratios (i.e., relative entropies) are restricted or penalized. The decision maker believes that the data will be generated by an unknown member of one of these sets. When relative entropies are constrained to be small, the decision maker believes that his model is a good approximation. The decision maker wants robustness against these alternatives because, as Anderson et al. (2003) emphasize, perturbations with small relative entropies are statistically difficult to distinguish from the approximating model.

Since the decision maker does not know the hidden Markov state, he is also compelled to weight the alternative conditional models. This paper assumes that at each date the appropriate summary of past signals about the hidden state is the decision maker's posterior under the approximating model, just as it is when the decision maker trusts his model. He makes a robust adjustment to these probabilities to accommodate the fact that they were constructed from the approximating model. As we shall see, the decision maker is not required to respect distortions to the distribution of today's hidden state that were implied by his decision making process at earlier dates. Hansen and Sargent (2005) studied a closely related decision problem that requires today's decision maker to commit to those prior distortions of the distribution of today's hidden states.

Section 2 formulates a Markov control problem in which a decision maker with a trusted model receives signals about hidden state variables. Subsequent sections view the model of section 2 as an approximation, use relative entropy to define a cloud of models that are

\footnotetext{
${ }^{2}$ See Weiland (2005).

${ }^{3}$ Alan Blinder, Robert E. Lucas, and Martin Feldstein have all argued against experimenting to refine model selection. See Cogley et al. (2005a) for quotations and further discussion.

${ }^{4}$ See Brock et al. $(2003,2004)$ for an approach to model selection and decision making that also emphasizes robustness.
} 
difficult to distinguish from it statistically, and construct a sequence of decision rules that can work well for all of those models. Section 3 uses results of Hansen and Sargent (2005) to represent distortions of an approximating model in terms of martingales defined on the same probability space as the approximating model. Section 4 then defines two operators, $\mathrm{T}^{1}$ and $\mathbf{T}^{2}$, respectively, that are indexed by penalty parameters $\left(\theta_{1}, \theta_{2}\right)$. In section 5 , we use $\mathrm{T}^{1}$ to adjust continuation values for concerns about model misspecification, conditioned on knowledge of the hidden state. We use $\mathrm{T}^{2}$ to adjust continuation values for concern about misspecification of the distribution of the hidden state. We interpret $\theta_{1}$ and $\theta_{2}$ as penalties on pertinent entropy terms. Hansen and Sargent (2006b) specializes our section 5 recursions to compute robust decision rules for the linear quadratic Gaussian case.

Section 6 relates the special $\theta_{1}=\theta_{2}$ case to a decision problem under commitment that we analyzed in Hansen and Sargent (2005) (they coincide when the discount factor is unity). We discuss the dynamic consistency of worst case beliefs about the hidden state in subsections 6.4 and 6.6. To prepare the machinery needed to construct stochastic discount factors like those mentioned in section 10, section 7 describes the worst case distribution over signals. Section 8 interprets our formulation and suggests modifications of it in terms of the multiple priors models of Epstein and Schneider (2003a,b). Section 9 relates our formulation to papers about reducing compound lotteries. Section 10 briefly describes a model of Hansen and Sargent (2006a) that combines special cases of the recursions in Hansen and Sargent (2006b) and the logarithmic preference specification of Tallarini (2000). Hansen and Sargent (2006a) construct the value function for a representative consumer who lives in a pure endowment economy and is unsure about the specifications of two submodels that might govern consumption growth, as well as being unsure about the probability for mixing those submodels. They deduce a multiplicative adjustment to the market price of risk that is contributed by the representative consumer's concerns about robustness. This model illustrates how the consumer's concern about misspecification of the probabilities for mixing submodels and also of the conditional means and other hidden components of consumption growth within each submodel can increase the volatility of the stochastic discount factor and enhance what are typically interpreted as risk premia. ${ }^{5}$ Section 11 concludes. Hansen and Sargent (2005) contains an extensive account of related literatures. An application to a decision problem with experimentation and learning about multiple submodels appears in Cogley et al. (2005a).

\section{A control problem without model uncertainty}

For $t \geq 0$, we partition a state vector as $x_{t}=\left[\begin{array}{l}y_{t} \\ z_{t}\end{array}\right]$, where $y_{t}$ is observed and $z_{t}$ is not. A vector $s_{t}$ of observable signals is correlated with the hidden state $z_{t}$ and is used by the decision maker to form beliefs about the hidden state. Let $Z$ denote a space of admissible unobserved states, $\mathcal{Z}$ a corresponding sigma algebra of subsets of states, and $\lambda$ a measure

\footnotetext{
${ }^{5}$ With enough data, the consumer can 'learn his way out of' concerns about misspecification on mixture probabilities across models and on parameter estimates because eventually the mixture probabilities will concentrate on one model and the parameter estimates will converge. But as we shall see, convergence does not come close to occurring within the sample for the quarterly record of post WWII U.S. consumption growth rates that we study.
} 
on the measurable space of hidden states $(Z, \mathcal{Z})$. Let $S$ denote the space of signals, $\mathcal{S}$ a corresponding sigma algebra, and $\eta$ a measure on the measurable space $(S, \mathcal{S})$ of signals.

Let $\left\{\mathcal{S}_{t}: t \geq 0\right\}$ denote a filtration, where $\mathcal{S}_{t}$ is generated by $y_{0}, s_{1}, \ldots, s_{t}$, where we shall assume that $s_{1}, \ldots, s_{t}$ is generated by system $\left.(1),(2), 3\right)$ below. We can apply Bayes' rule to $\tau$ to deduce a density $q_{t}$, relative to the measure $\lambda$, for $z_{t}$ conditioned on information $\mathcal{S}_{t}$. Let $\left\{\mathcal{X}_{t}: t \geq 0\right\}$ be a larger filtration where $\mathcal{X}_{t}$ is generated by $x_{0}, w_{1}, w_{2}, \ldots, w_{t}$. The smallest sigma algebra generated by all states for $t \geq 0$ is $\mathcal{X}_{\infty} \doteq \bigvee_{t \geq 0} \mathcal{X}_{t}$; the smallest sigma algebra generated by all signals for $t \geq 0$ is $\mathcal{S}_{\infty} \doteq \bigvee_{t>0} \mathcal{S}_{t}$. Let $A$ denote a feasible set of actions, which we take to be a Borel set of some finite dimensional Euclidean space, and let $\mathcal{A}_{t}$ be the set of $A$-valued random vectors that are $\mathcal{S}_{t}$ measurable. ${ }^{6}$

Signals and states are determined by the transition functions

$$
\begin{aligned}
y_{t+1} & =\pi_{y}\left(s_{t+1}, y_{t}, a_{t}\right) \\
z_{t+1} & =\pi_{z}\left(x_{t}, a_{t}, w_{t+1}\right) \\
s_{t+1} & =\pi_{s}\left(x_{t}, a_{t}, w_{t+1}\right)
\end{aligned}
$$

where $\left\{w_{t+1}: t \geq 0\right\}$ is an i.i.d. sequence of random variables. Knowledge of $y_{0}$ and $\pi_{y}$ allows us to construct $y_{t}$ recursively from signals and actions. The construction of $x_{t}$ in equation (1) - (2) and the informational constraint on action processes imply that $x_{t}$ is $\mathcal{X}_{t}$ measurable and $y_{t}$ is $\mathcal{S}_{t}$ measurable. Substituting (3) into (1) gives:

$$
y_{t+1}=\pi_{y}\left[\pi_{s}\left(x_{t}, a_{t}, w_{t+1}\right), y_{t}, a_{t}\right] \doteq \bar{\pi}_{y}\left(x_{t}, a_{t}, w_{t+1}\right) .
$$

Equations (2) and (3) determine a conditional density $\tau\left(z_{t+1}, s_{t+1} \mid x_{t}, a_{t}\right)$ relative to the product measure $\lambda \times \eta$.

As a benchmark, consider the following decision problem under complete confidence in model (1), (2), (3) but incomplete information about the state:

Problem 2.1.

$$
\max _{a_{t} \in \mathcal{A}_{t}: t \geq 0} E\left[\sum_{t=0}^{T} \beta^{t} U\left(x_{t}, a_{t}\right) \mid \mathcal{S}_{0}\right], \quad \beta \in(0,1)
$$

subject to (1), (2), and (3).

To make problem 2.1 recursive, let ${ }^{*}$ denote a next period value and use $\tau$ to construct two densities for the signal:

$$
\begin{aligned}
\kappa\left(s^{*} \mid y_{t}, z_{t}, a_{t}\right) & \doteq \int \tau\left(z^{*}, s^{*} \mid y_{t}, z_{t}, a_{t}\right) d \lambda\left(z^{*}\right) \\
\varsigma\left(s^{*} \mid y_{t}, q_{t}, a_{t}\right) & \doteq \int \kappa\left(s^{*} \mid y_{t}, z, a_{t}\right) q_{t}(z) d \lambda(z)
\end{aligned}
$$

By Bayes' rule,

$$
q_{t+1}\left(z^{*}\right)=\frac{\int \tau\left(z^{*}, s_{t+1} \mid y_{t}, z, a_{t}\right) q_{t}(z) d \lambda(z)}{\varsigma\left(s_{t+1} \mid y_{t}, q_{t}, a_{t}\right)} \equiv \pi_{q}\left(s_{t+1}, y_{t}, q_{t}, a_{t}\right) .
$$

\footnotetext{
${ }^{6}$ We could easily allow $A$ to depend on the observable component of the state.
} 
In particular applications, $\pi_{q}$ can be computed with methods that specialize Bayes' rule (e.g., the Kalman filter or a discrete time version of the Wonham (1964) filter).

Take $\left(y_{t}, q_{t}\right)$ as the state for a recursive formulation of problem 2.1. The transition law is (1) and (5). Let $\pi=\left[\begin{array}{l}\pi_{y} \\ \pi_{q}\end{array}\right]$. Then we can rewrite problem 2.1 in the alternative form:

Problem 2.2. Choose a sequence of decision rules for $a_{t}$ as functions of $\left(y_{t}, q_{t}\right)$ for each $t \geq 0$ that maximize

$$
E\left[\sum_{t=0}^{T} \beta^{t} U\left(x_{t}, a_{t}\right) \mid \mathcal{S}_{0}\right]
$$

subject to (1), (5), a given density $q_{0}(z)$, and the density $\kappa\left(s_{t+1} \mid y_{t}, z_{t}, a_{t}\right)$. The Bellman equation for this problem is

$$
W(y, q)=\max _{a \in A} \int\left\{U(x, a)+\beta \int W^{*}\left[\pi\left(s^{*}, y, q, a\right)\right] \kappa\left(s^{*} \mid y, z, a\right) d \eta\left(s^{*}\right)\right\} q(z) d \lambda(z) .
$$

In an infinite horizon version of problem $2.2, W^{*}=W$.

\section{$2.1 \quad$ Examples}

Examples of problem 2.2 in economics include Jovanovic (1979), Jovanovic (1982), Jovanovic and Nyarko (1995, 1996), and Bergemann and Valimaki (1996). Examples from outside economics appear in Elliott et al. (1995). Problems that we are especially interested in are illustrated in the following four examples. (More examples appear in section 10.)

Example 2.3. Model Uncertainty I: two submodels. Let the hidden state $z \in\{0,1\}$ index one of two submodels. Let

$$
\begin{aligned}
y_{t+1} & =s_{t+1} \\
z_{t+1} & =z_{t} \\
s_{t+1} & =\pi_{s}\left(y_{t}, z, a_{t}, w_{t+1}\right) .
\end{aligned}
$$

The hidden state is time invariant. The decision maker has prior probability $\operatorname{Prob}(z=0)=$ q. The third equation in (7) depicts two laws of motion. Cogley et al. (2005a) and Cogley et al. (2005b) study the value of monetary policy experimentation in a model in which a is an inflation target and $\pi_{s}(y, z, a, w)=\bar{\pi}_{y}(y, z, a, w)$ for $z \in\{0,1\}$ represent two submodels of inflation-unemployment dynamics.

Example 2.4. Model Uncertainty II: a continuum of submodels. The observable state y takes two possible values $\left\{y_{L}, y_{H}\right\}$. Transition dynamics are still described by (7), but now there is a continuum of models indexed by the hidden state $z \in[0,1] \times[0,1]$ that stands for unknown values of two transition probabilities for an observed state variable $y$. Given z, we can use the third equation of (7) to represent a two state Markov chain that governs the observable state $y$ (see Elliott et al. (1995)), $P=\left[\begin{array}{cc}p_{11} & 1-p_{11} \\ 1-p_{22} & p_{22}\end{array}\right]$, where $\left(p_{11}, p_{22}\right)=z$. The decision maker has prior distribution $g_{0,1}\left(p_{11}\right) g_{0,2}\left(p_{22}\right)$ on $z ; g_{0,1}$ and $g_{0,2}$ are beta distributions. 
Example 2.5. Model Uncertainty III: a components model of income dynamics with an unknown fixed effect in labor income. The utility function $U\left(a_{t}\right)$ is a concave function of consumption $a_{t}$; $y_{2 t}$ is the level of financial assets, and $y_{1 t}=s_{t}$ is observed labor income. The evolution equations are

$$
\begin{aligned}
y_{1, t+1} & =s_{t+1} \\
y_{2, t+1} & =R\left[y_{2, t}+y_{1, t}-a_{t}\right] \\
z_{1, t+1} & =z_{1, t} \\
z_{2, t+1} & =\rho z_{2, t}+\sigma_{1} w_{1, t+1} \\
s_{t+1} & =z_{1, t}+z_{2, t}+\sigma_{2} w_{2, t+1}
\end{aligned}
$$

where $w_{t+1} \sim \mathcal{N}(0, I)$ is an i.i.d. bivariate Gaussian process, $R \leq \beta^{-1}$ is a gross return on financial assets $y_{2, t},|\rho|<1, z_{1, t}$ is one unobserved constant component of labor income, and $z_{2, t}$ is another unobserved serially correlated component of labor income. A decision maker has a prior $q_{0}$ over $\left(z_{1,0}, z_{2,0}\right)$.

Example 2.6. Estimation of drifting coefficients regression model. The utility function $U\left(x_{t}, a_{t}\right)=-L\left(z_{t}-a_{t}\right)$, where $L$ is a loss function and $a_{t}$ is a time-t estimate of the coefficient vector $z_{t}$. The evolution equation is

$$
\begin{aligned}
y_{t+1} & =s_{t+1} \\
z_{t+1} & =\rho z_{t}+\sigma_{1} w_{1, t+1} \\
s_{t+1} & =y_{t} \cdot z_{t}+\sigma_{2} w_{2, t+1}
\end{aligned}
$$

where $w_{t+1} \sim \mathcal{N}(0, I)$ and there is a prior $q_{0}(z)$ on an initial set of coefficients.

\subsection{Modified problems that distrust $\kappa\left(s^{*} \mid y, z, a\right)$ and $q(z)$}

This paper studies modifications of problem 2.2 in which the decision maker wants a decision rule that is robust to possible misspecifications of equations (1)-(2). We express the Bellman equation as (6) and use the decomposition of $\varsigma$ in (4). Representation (6) focuses the decision maker's concerns on two aspects of the stochastic structure: the conditional distribution of next period's signals $\kappa\left(s^{*} \mid y, z, a\right)$ and the distribution over this period's value of the hidden state $q(z)$. We propose a recursive formulation of a robust control and estimation problem that allows a decision maker to doubt either or both of these aspects of his stochastic specification. To prepare the way, section 3 describes how misspecifications of the decision maker's approximating model can be represented in terms of sequences of nonnegative random variables that form martingales under that approximating model.

\section{Using martingales to represent model misspecifica- tions}

Hansen and Sargent (2005) use a nonnegative $\mathcal{X}_{t}$-measurable function $M_{t}$ with $E M_{t}=1$ to create a distorted probability measure that is absolutely continuous with respect to the 
probability measure over $\mathcal{X}_{t}$ generated by the model (1) - (2). The random variable $M_{t}$ is a martingale under this probability measure. Using $M_{t}$ as a Radon-Nikodym derivative generates a distorted measure under which the expectation of a bounded $\mathcal{X}_{t}$-measurable random variable $W_{t}$ is $\tilde{E} W_{t} \doteq E M_{t} W_{t}$. The entropy of the distortion at time $t$ conditioned on date zero information is $E\left(M_{t} \log M_{t} \mid \mathcal{X}_{0}\right)$ or $E\left(M_{t} \log M_{t} \mid \mathcal{S}_{0}\right)$.

\subsection{Recursive representations of distortions}

We often factor a density $F_{t+1}$ for an $\mathcal{X}_{t+1}$-measurable random variable as $F_{t+1}=F_{t} f_{t+1}$ where $f_{t+1}$ is a one-step ahead density conditioned on $\mathcal{X}_{t}$. It is also useful to factor $M_{t}$. Thus, take a nonnegative martingale $\left\{M_{t}: t \geq 0\right\}$ and form

$$
m_{t+1}=\left\{\begin{array}{ccc}
\frac{M_{t+1}}{M_{t}} & \text { if } & M_{t}>0 \\
1 & \text { if } & M_{t}=0
\end{array}\right.
$$

Then $M_{t+1}=m_{t+1} M_{t}$ and

$$
M_{t}=M_{0} \prod_{j=1}^{t} m_{j}
$$

The random variable $M_{0}$ has unconditional expectation equal to unity. By construction, $m_{t+1}$ has date $t$ conditional expectation equal to unity. For a bounded random variable $W_{t+1}$ that is $\mathcal{X}_{t+1}$-measurable, the distorted conditional expectation implied by the martingale $\left\{M_{t}: t \geq 0\right\}$ is

$$
\frac{E\left(M_{t+1} W_{t+1} \mid \mathcal{X}_{t}\right)}{E\left(M_{t+1} \mid \mathcal{X}_{t}\right)}=\frac{E\left(M_{t+1} W_{t+1} \mid \mathcal{X}_{t}\right)}{M_{t}}=E\left(m_{t+1} W_{t+1} \mid \mathcal{X}_{t}\right)
$$

provided that $M_{t}>0$. We use $m_{t+1}$ to represent distortions of the conditional probability distribution for $\mathcal{X}_{t+1}$ given $\mathcal{X}_{t}$. For each $t \geq 0$, construct the space $\mathcal{M}_{t+1}$ of all nonnegative, $\mathcal{X}_{t+1}$-measurable random variables $m_{t+1}$ for which $E\left(m_{t+1} \mid \mathcal{X}_{t}\right)=1$.

The conditional (on $\mathcal{X}_{t}$ ) relative entropy of a nonnegative random variable $m_{t+1}$ in $\mathcal{M}_{t+1}$ is $\varepsilon_{t}^{1}\left(m_{t+1}\right) \doteq E\left(m_{t+1} \log m_{t+1} \mid \mathcal{X}_{t}\right)$.

\subsection{Distorting likelihoods with hidden information}

The random variable $M_{t}$ is adapted to $\mathcal{X}_{t}$ and is a likelihood ratio for two probability distributions over $\mathcal{X}_{t}$. The $\mathcal{S}_{t}$-measurable random variable $G_{t}=E\left(M_{t} \mid \mathcal{S}_{t}\right)$ implies a likelihood ratio for the reduced information set $\mathcal{S}_{t} ; G_{t}$ assigns distorted expectations to $\mathcal{S}_{t}$-measurable random variables that agree with $M_{t}$, and $\left\{G_{t}: t \geq 0\right\}$ is a martingale adapted to $\left\{\mathcal{S}_{t}: t \geq 0\right\}$.

Define the $\mathcal{X}_{t}$-measurable random variable $h_{t}$ by

$$
h_{t} \doteq\left\{\begin{array}{ccc}
\frac{M_{t}}{E\left(M_{t} \mid \mathcal{S}_{t}\right)} & \text { if } & E\left(M_{t} \mid \mathcal{S}_{t}\right)>0 \\
1 & \text { if } & E\left(M_{t} \mid \mathcal{S}_{t}\right)=0
\end{array}\right.
$$

and decompose $M_{t}$ as

$$
M_{t}=h_{t} G_{t} .
$$


Decompose entropy as

$$
\begin{aligned}
E\left(M_{t} \log M_{t} \mid \mathcal{S}_{0}\right) & =E\left[G_{t} h_{t}\left(\log h_{t}+\log G_{t}\right) \mid \mathcal{S}_{0}\right] \\
& =E\left(G_{t} h_{t} \log h_{t} \mid \mathcal{S}_{0}\right)+E\left(G_{t} \log G_{t} \mid \mathcal{S}_{0}\right)
\end{aligned}
$$

where we have dropped an $h_{t}$ from the last term because $E\left(h_{t} \mid \mathcal{S}_{t}\right)=1$ and $G_{t}$ is $\mathcal{S}_{t}$ measurable. Define $\varepsilon_{t}^{2}\left(h_{t}\right) \doteq E\left(h_{t} \log h_{t} \mid \mathcal{S}_{t}\right)$ as the conditional (on $\mathcal{S}_{t}$ ) relative entropy.

We now have the tools to represent and measure misspecifications of the two components $\kappa\left(s^{*} \mid y, z, a\right)$ and $q(z)$ in (6). In (9), $M_{t}$ distorts the probability distribution of $\mathcal{X}_{t}, h_{t}$ distorts the probability of $\mathcal{X}_{t}$ conditioned on $\mathcal{S}_{t}, G_{t}$ distorts the probability of $\mathcal{S}_{t}$, and $m_{t+1}$ distorts the probability of $\mathcal{X}_{t+1}$ given $\mathcal{X}_{t}$. We use multiplication by $m_{t+1}$ to distort $\kappa$ and multiplication by $h_{t}$ to distort $q$. We use $\epsilon_{t}^{1}\left(m_{t+1}\right)$ to measure $m_{t+1}$ and $\epsilon_{t}^{2}\left(h_{t}\right)$ to measure $h_{t}$.

\section{Two pairs of operators}

This section introduces two pairs of risk-sensitivity operators, $\left(R_{t}^{1}, T^{1}\right)$ and $\left(R_{t}^{2}, T^{2}\right)$. In section 5, we use the $\mathrm{T}^{1}$ and $\mathrm{T}^{2}$ operators to define recursions that induce robust decision rules.

\section{1 $\mathrm{R}_{t}^{1}$ and $\mathrm{T}^{1}$}

For $\theta>0$, let $V_{t+1}$ be an $\mathcal{X}_{t+1}$-measurable random variable for which $E\left[\exp \left(-\frac{V_{t+1}}{\theta}\right) \mid \mathcal{X}_{t}\right]<$ $\infty$. Then define

$$
\begin{aligned}
\mathrm{R}_{t}^{1}\left(V_{t+1} \mid \theta\right) & =\min _{m_{t+1} \in \mathcal{M}_{t+1}} E\left(m_{t+1} V_{t+1} \mid \mathcal{X}_{t}\right)+\theta \varepsilon_{t}^{1}\left(m_{t+1}\right) \\
& =-\theta \log E\left[\exp \left(-\frac{V_{t+1}}{\theta}\right) \mid \mathcal{X}_{t}\right]
\end{aligned}
$$

The minimizing choice of $m_{t+1}$ is

$$
m_{t+1}^{*}=\frac{\exp \left(-\frac{V_{t+1}}{\theta}\right)}{E\left[\exp \left(-\frac{V_{t+1}}{\theta}\right) \mid \mathcal{X}_{t}\right]}
$$

where the term in the denominator assures that $E m_{t+1}^{*} \mid \mathcal{X}_{t}=1$.

In the limiting $\theta=\infty$ case, $\mathrm{R}_{t}^{1}\left(V_{t+1} \mid \infty\right)=E\left(V_{t+1} \mid \mathcal{X}_{t}\right)$. Notice that this expectation can depend on the hidden state. When $\theta<\infty$, R $\mathrm{R}_{t}^{1}$ adjusts $E\left(V_{t+1} \mid \mathcal{X}_{t}\right)$ by using a worst-case belief about the probability distribution of $\mathcal{X}_{t+1}$ conditioned on $\mathcal{X}_{t}$ that is implied by the twisting factor (11), as well as adding an entropy penalty. When the conditional moment restriction $E\left[\exp \left(-\frac{V_{t+1}}{\theta}\right) \mid \mathcal{X}_{t}\right]<\infty$ is not satisfied, we define $\mathrm{R}_{\mathrm{t}}^{1}$ to be $-\infty$ on the relevant conditioning events.

When the $\mathcal{X}_{t+1}$-measurable random variable $V_{t+1}$ takes the special form $W\left(y_{t+1}, q_{t+1}, z_{t+1}\right)$, the $\mathrm{R}_{t}^{1}(\cdot \mid \theta)$ operator implies another operator:

$\mathrm{T}^{1}(W \mid \theta)(y, q, z, a)=-\theta \log \int \exp \left(-\frac{W\left[\pi\left(s^{*}, y, q, a\right), z^{*}\right]}{\theta}\right) \tau\left(z^{*}, s^{*} \mid y, z, a\right) d \lambda\left(z^{*}\right) d \eta\left(s^{*}\right)$. 
The transformation $\mathrm{T}^{1}$ maps a value function that depends on next period's state $\left(y^{*}, q^{*}, z^{*}\right)$ into a risk-adjusted value function that depends on $(y, q, z, a)$. Associated with this risk sensitivity adjustment $\mathrm{T}^{1}$ is a worst-case distortion in the transition dynamics for the state and signal process. Let $\phi$ denote a nonnegative density function defined over $\left(z^{*}, s^{*}\right)$ satisfying

$$
\int \phi\left(z^{*}, s^{*}\right) \tau\left(z^{*}, s^{*} \mid y, z, a\right) d \lambda\left(z^{*}\right) d \eta\left(s^{*}\right)=1
$$

The corresponding entropy measure is:

$$
\int \log \left[\phi\left(z^{*}, s^{*}\right)\right] \phi\left(z^{*}, s^{*}\right) \tau\left(z^{*}, s^{*} \mid y, z, a\right) d \lambda\left(z^{*}\right) d \eta\left(s^{*}\right) .
$$

In our recursive formulation, we think of $\phi$ as a possibly infinite dimensional control vector (a density function) and consider the minimization problem:

$$
\min _{\phi \geq 0} \int\left(W\left[\pi\left(s^{*}, y, q, a\right), z^{*}\right]+\theta \log \left[\phi\left(z^{*}, s^{*}\right)\right]\right) \phi\left(z^{*}, s^{*}\right) \tau\left(z^{*}, s^{*} \mid y, z, a\right) d \lambda\left(z^{*}\right) d \eta\left(s^{*}\right)
$$

subject to (12). The associated worst-case density conditioned on $\mathcal{X}_{t}$ is $\phi_{t}\left(z^{*}, s^{*}\right) \tau\left(z^{*}, s^{*} \mid x_{t}, a_{t}\right)$ where

$$
\phi_{t}\left(z^{*}, s^{*}\right)=\frac{\exp \left(-\frac{V\left[\pi\left(s^{*}, y_{t}, q_{t}, a_{t}\right), z^{*}\right]}{\theta}\right)}{E\left[\exp \left(-\frac{V\left[\pi\left(s_{t+1}, y_{t}, q_{t}, a_{t}\right), z_{t+1}\right]}{\theta}\right) \mid \mathcal{X}_{t}\right]} .
$$

\section{2 $\mathrm{R}_{t}^{2}$ and $\mathrm{T}^{2}$}

For $\theta>0$, let $\hat{W}_{t}$ be an $\mathcal{X}_{t}$-measurable function for which $E\left[\exp \left(-\frac{\hat{W}_{t}}{\theta}\right) \mid \mathcal{S}_{t}\right]<\infty$. Then define

$$
\begin{aligned}
\mathrm{R}_{t}^{2}\left(\hat{V}_{t} \mid \theta\right) & =\min _{h_{t} \in \mathcal{H}_{t}} E\left(h_{t} \hat{V}_{t} \mid \mathcal{S}_{t}\right)+\theta \varepsilon_{t}^{2}\left(h_{t}\right) \\
& =-\theta \log E\left[\exp \left(-\frac{\hat{V}_{t}}{\theta}\right) \mid \mathcal{S}_{t}\right]
\end{aligned}
$$

The minimizing choice of $h_{t}$ is

$$
h_{t}^{*}=\frac{\exp \left(-\frac{\hat{W}_{t}}{\theta}\right)}{E\left[\exp \left(-\frac{\hat{W}_{t}}{\theta}\right) \mid \mathcal{S}_{t}\right]}
$$

where the term in the denominator assures that $E h_{t}^{*} \mid \mathcal{S}_{t}=1$.

When an $\mathcal{X}_{t}$-measurable function has the special form $\hat{W}_{t}=\hat{V}\left(y_{t}, q_{t}, z_{t}, a_{t}\right)$, (14) implies another operator

$$
\mathrm{\top}^{2}(\hat{V} \mid \theta)(y, q, a)=-\theta \log \int \exp \left[-\frac{\hat{V}(y, q, z, a)}{\theta}\right] q(z) d \lambda(z) .
$$


The associated minimization problem is:

$$
\min _{\psi \geq 0} \int[\hat{V}(y, q, z, a)+\theta \log \psi(z)] \psi(z) q(z) d \lambda(z)
$$

subject to $(15)$, where $\psi(z)$ is a relative density that satisfies:

$$
\int \psi(z) q(z) d \lambda(z)=1
$$

and the entropy measure is

$$
\int[\log \psi(z)] \psi(z) q(z) d \lambda(z)
$$

The optimized density conditioned on $\mathcal{S}_{t}$ is $\psi_{t}(z) q_{t}(z)$, where

$$
\psi_{t}(z)=\frac{\exp \left(-\frac{\hat{V}\left(y_{t}, q_{t}, z, a_{t}\right)}{\theta}\right)}{E\left[\exp \left(-\frac{\hat{V}\left(y_{t}, q_{t}, z, a_{t}\right)}{\theta}\right) \mid \mathcal{S}_{t}\right]}
$$

\section{Control problems with model uncertainty}

We propose robust control problems that take $q_{t}(z)$ as the component of the decision maker's state vector that summarizes the history of signals. The decision maker's model includes the law of motion (5) for $q$ (Bayes' law) under the approximating model (1), (2), (3). Two

recursions that generalize Bellman equation (6) express alternative views about the decision maker's fear of misspecification. A first recursion works with value functions that include the hidden state $z$ as a state variable. Let

$$
\check{W}(y, q, z)=U(x, a)+E\left\{\beta \check{W}^{*}\left[\pi\left(s^{*}, y, q, a\right), z^{*}\right] \mid x, q\right\},
$$

where the action $a$ solves:

$$
W(y, q)=\max _{a} E\left[U(x, a)+E\left\{\beta \check{W}^{*}\left[\pi\left(s^{*}, y, q, a\right), z^{*}\right] \mid x, q, a\right\} \mid y, q, a\right] .
$$

The value function $\check{W}$ depends on the hidden state $z$, whereas the value function $W$ in (6) does not. A second recursion modifies the ordinary Bellman equation (6), which we can express as:

$$
W(y, q)=\max _{a} E\left[U(x, a)+E\left\{\beta W^{*}\left[\pi\left(s^{*}, y, q, a\right)\right] \mid x, q, a\right\} \mid y, q, a\right] .
$$

Although they use different value functions, without concerns about model misspecification, formulations (17)-(18) and (19) imply identical control laws. Furthermore, a $W(y, q)$ that satisfies (19) also obeys (18) by virtue of the law of iterated expectations. Because Bellman equation (19) is computationally more convenient, the pair (17)-(18) is not used in the standard problem without a concern for robustness. However, with a concern about robustness, 
a counterpart to (17)-(18) becomes useful when the decision maker wants to explore distortions of the joint conditional distribution $\tau\left(s^{*}, z^{*} \mid y, z, a\right) \cdot{ }^{7}$ Distinct formulations emerge from (18) and (19) when we replace the conditional expectation $E(\cdot \mid y, q, a)$ with $\mathrm{T}^{2}\left(\cdot \mid \theta_{2}\right)$ and the conditional expectation $E(\cdot \mid x, q, a)$ with $\mathrm{T}^{1}\left(\cdot \mid \theta_{1}\right)$. When $\theta_{1}=\theta_{2}=+\infty$, (17)-(18) or (19) lead to value functions and decision rules equivalent to those from (6). When $\theta_{1}<+\infty$ and $\theta_{2}<+\infty$, recursions (17)-(18) and (19) lead to different decision rules because they take different views about the conditional distributions that the malevolent player wants to distort, or equivalently, about the aspects of the stochastic specification in the approximating model against which the decision maker seeks robustness.

\subsubsection{Which conditional distributions to distort?}

The approximating model (1), (2), (3) makes both tomorrow's signal $s^{*}$ and tomorrow's state $z^{*}$ functions of $x$. When tomorrow's value function depends on $s^{*}$ but not on $z^{*}$ as in (19), the minimizing player chooses to distort only $\kappa\left(s^{*} \mid y, z, a\right)$, which amounts to being concerned about misspecifications of the evolution equation (3) for the signal and not (2) for the hidden state. Such a continuation value function imparts no additional incentive to distort the evolution equation (2) of $z^{*}$ conditioned on $s^{*}$ and $x .8$ Such a continuation value that depends on $s^{*}$ but not on $z^{*}$ thus imparts concerns about a limited array of distortions that ignore possible misspecification of the $z^{*}$ evolution (2). Therefore, when we want to direct the maximizing agent's concerns about misspecification onto the conditional distribution $\kappa\left(s^{*} \mid y, z, a\right)$, we should form a current period value that depends only on the history of the signal and of the observed state. We do this in recursion (23) below.

However, in some situations, we might want to extend the maximizing player's concerns about misspecification to the joint distribution $\tau\left(z^{*}, s^{*} \mid y, z, a\right)$ of $z^{*}$ and $s^{*}$. We can do this by making tomorrow's value function for the minimizing player also depend on $z^{*}$. In recursions (20)-(21) below, we form a continuation value function that depends on $z^{*}$ and thereby extend recursions (17), (18) to incorporate concerns about misspecification of (2).

Thus, (20)-(21) below will induce the minimizing player to distort the distribution of $z^{*}$ conditional on $\left(s^{*}, x, a\right)$, while the formulation in (23) will not.

\section{$5.1 \quad$ Value function depends on $(x, q)$}

By defining a value function that depends on the hidden state, we focus the decision maker's attention on misspecification of the joint conditional distribution $\tau\left(z^{*}, s^{*} \mid y, z, a\right)$ of $\left(s^{*}, z^{*}\right)$. We modify recursions (17)-(18) by updating a value function according to

$$
W(y, q, z)=U(x, a)+\mathrm{T}^{1}\left[\beta W^{*}\left(y^{*}, q^{*}, z^{*}\right) \mid \theta_{1}\right](x, q, a)
$$

after choosing an action according to

$$
\max _{a} \mathrm{~T}^{2}\left(U(x, a)+\mathrm{T}^{1}\left[\beta W^{*}\left(y^{*}, q^{*}, z^{*}\right) \mid \theta_{1}\right](x, q, a) \mid \theta_{2}\right)(y, q, a),
$$

\footnotetext{
${ }^{7}$ Another way to express his concerns is that in this case the decision maker fears that (2) and (3) are both misspecified.

${ }^{8}$ Dependence between $\left(s^{*}, z^{*}\right)$ conditioned on $x$ under the approximating model means that in the process of distorting $s^{*}$ conditioned on $(x, a)$, the minimizing player may indirectly distort the distribution of $z^{*}$ conditioned on $(x, a)$. But he does not distort the distribution of $z^{*}$ conditioned on $\left(s^{*}, x, a\right)$
} 
for $\theta_{1} \geq \underline{\theta}_{1}, \theta_{2} \geq \underline{\theta}_{2}\left(\theta_{1}\right)$ for $\underline{\theta}_{1}, \underline{\theta}_{2}$ that make the problems well posed. ${ }^{9}$ Updating the value function by recursion (20) makes it depend on $(x, q)$, while using (21) to guide decisions makes actions depend only on the observable state $(y, q)$. Thus, continuation value $\breve{W}$ depends on unobserved states, but actions do not. To retain the dependence of the continuation value on $z$, (20) refrains from using the $\mathrm{T}^{2}$ transformation when up-dating continuation values. The fixed point of (20)-(21) is the value function for an infinite horizon problem. For the finite horizon counterpart, we begin with a terminal value function and view the right side of (20) as mapping next period's value function into the current period value function.

\subsubsection{Time inconsistency of maximizing player's preferences}

In formulation (20)-(21), the current period decision maker acknowledges the dependence on the current hidden state of discounted future returns. For simplicity, suppose that we set $\theta_{1}=\infty$. Then $W(y, q, z)$ gives the discounted value of an objective conditioned on the hidden state $z$. That this hidden state helps predict future signals and future observable state vectors is reflected the dependence of this value function on $z$. This dependence remains when we let $\theta_{1}<\infty$, thus activating a concern about model misspecification conditioned on the current period value of the state $z$. Such dependence is also present in a commitment formulation of the problem discussed in Hansen and Sargent (2005). In the present formulation without commitment, we use recursion (20) to portray a Markov perfect equilibrium of a game in which the date $t$ maximizing decision maker (and his malevolent companions) take as given the decisions of future maximizing decision makers (and their malevolent companions). ${ }^{10}$

That the $\mathrm{T}^{2}$ operator is applied only at the last stage of the backward induction in (20)(21) renders the preferences of the time 0 agent dynamically inconsistent. ${ }^{11}$ The dynamic inconsistency reflects a conflict between the interests of decision makers at different times, one that vanishes when $\beta \rightarrow 1$ and which we now describe.

To explore the preferences implicit in this formulation it is convenient to apply the operators $\mathrm{R}_{t}^{1}$ and $\mathrm{R}_{t}^{2}$ to continuation values. Let $V_{t+1}$ denote the continuation values of a stochastic process of actions from date $t+1$ forward. This continuation value can depend on the future states. It is $\mathcal{X}_{t+1}$ measurable but not necessarily $\mathcal{S}_{t+1}$ measurable. Assess this action process at date $t+1$ using $\mathrm{R}_{t+1}^{2}\left(V_{t+1} \mid \theta_{2}\right)$, which makes a robust adjustment and results in an $\mathcal{S}_{t+1}$ measurable continuation value.

Consider two such continuation values, $V_{t+1}^{a}$ and $V_{t+1}^{b}$, where

$$
\mathrm{R}_{t+1}^{2}\left(V_{t+1}^{a} \mid \theta_{2}\right) \geq \mathrm{R}_{t+1}^{2}\left(V_{t+1}^{b} \mid \theta_{2}\right) .
$$

We are interested in a date $t$ ranking of these two after we discount (22) and add a common current period contribution $U_{t}$ to both before applying $\mathrm{R}_{t}^{2}$. This results in two continuation values that are not necessarily comparable, namely, $U_{t}+\mathrm{R}_{t}^{1}\left(\beta V_{t+1}^{a} \mid \theta_{1}\right)$ and $U_{t}+\mathrm{R}_{t}^{1}\left(\beta V_{t+1}^{b} \mid \theta_{1}\right)$. For some realized signal histories, the ranking in inequality (22) can be reversed, even after applying $\mathrm{R}_{t}^{2}$.

\footnotetext{
${ }^{9}$ Limits on $\theta_{1}$ and $\theta_{2}$ are typically needed to make the outcomes of the $\mathrm{T}^{1}$ and $\mathrm{T}^{2}$ operators be finite.

${ }^{10}$ Laibson (1997) uses a Markov perfect equilibrium of such a game to model the decisions of made by someone with intertemporally inconsistent preferences coming from hyperbolic discounting.

${ }^{11}$ That dynamic inconsistency is what prompts us to model decisions as the Markov perfect equilibrium represented in recursion (20).
} 
It is instructive to consider the special case in which $U_{t}$ is $\mathcal{S}_{t}$ measurable. Then

$$
\mathrm{R}_{t}^{2}\left[U_{t}+\mathrm{R}_{t}^{1}\left(\beta V_{t+1}^{j} \mid \theta_{1}\right) \mid \theta_{2}\right]=U_{t}+\mathrm{R}_{t}^{2}\left[\mathrm{R}_{t+1}^{1}\left(\beta V_{t+1}^{j} \mid \theta_{1}\right) \mid \theta_{2}\right]
$$

for $j=a, b$. The source of possible intertemporal reversal of rankings is that inequality (22) does not imply:

$$
\mathrm{R}_{t}^{2}\left[\mathrm{R}_{t+1}^{1}\left(\beta V_{t+1}^{a} \mid \theta_{1}\right) \mid \theta_{2}\right] \geq \mathrm{R}_{t}^{2}\left[\mathrm{R}_{t+1}^{1}\left(\beta V_{t+1}^{b} \mid \theta_{1}\right) \mid \theta_{2}\right]
$$

If, however, we strengthen inequality (22) to be:

$$
V_{t+1}^{a} \geq V_{t+1}^{b}
$$

then the rankings are preserved. Thus, when we limit comparisons to ones conditioned on hidden states, then intertemporal inconsistency vanishes.

In the next subsection, we propose an alternative approach that avoids the conflict that is the source of this intertemporal inconsistency at the cost of giving the hidden states a less direct role. In particular, as we shall see, this alternative approach considers value functions that depend only on $(y, q)$ and not the hidden state $z$. This formulation removes an incentive to explore misspecification of the hidden state dynamics themselves and instead focuses only on how those misspecifications might affect the evolution of signals.

\subsection{Value function depends on $(y, q)$}

To focus on misspecifications of the conditional distribution $\kappa\left(s^{*} \mid y, z, a\right)$, we want the minimizing player's value function to depend only on the reduced information encoded in $(y, q)$. For this purpose, we use the following counterpart to recursion (19):

$$
W(y, q)=\max _{a} \mathrm{~T}^{2}\left(U(x, a)+\mathrm{T}^{1}\left[\beta W^{*}\left(y^{*}, q^{*}\right) \mid \theta_{1}\right](x, q, a) \mid \theta_{2}\right)(y, q, a)
$$

for $\theta_{1} \geq \underline{\theta}_{1}$ and $\theta_{2} \geq \underline{\theta}_{2}\left(\theta_{1}\right)$. Although $z^{*}$ is excluded from the value function $W^{*}, z$ may help predict the observable state $y^{*}$ or it may enter directly into the current period reward function, so application of the operator $\mathrm{T}^{1}$ creates a value function that depends on $(x, q, a)$, including the hidden state $z$. Since the malevolent agent observes $z$, he can distort the dynamics for the observable state conditioned on $z$ via the $\mathrm{T}^{1}$ operator. Subsequent application of $\mathrm{T}^{2}$ gives a value function that depends on $(y, q, a)$, but not $z ; \mathrm{T}^{2}$ distorts the hidden state distribution. The decision rule sets action $a$ as a function of $(y, q)$. The fixed point of Bellman equation (23) gives the value function for an infinite horizon problem. For finite horizon problems, we iterate on the mapping defined by the right side of (23), beginning with a known terminal value function. Recursion (23) extends the recursive formulation of risk-sensitivity with discounting advocated by Hansen and Sargent (1995) to situations with a hidden state.

\subsection{A third formulation that forgets that $z$ is hidden}

It is interesting to contrast the above approaches with an alternative one that is be feasible for problems in which $z$ does not appear directly in $U$ but instead either $y$ appears alone or 
$y$ and $q$ both appear. Then one could proceed by simply applying a single risk-sensitivity operator. For such problems, the Bellman equation without concerns about robustness (6) could also be expressed as

$$
W(y, q)=\max _{a \in A} U(y, q, a)+\beta \int W^{*}\left[\pi\left(s^{*}, y, q, a\right)\right] \varsigma\left(s^{*} \mid y, q, a\right) d \eta\left(s^{*}\right) .
$$

The analysis of robust control problems without hidden states in Hansen and Sargent (1995) and Hansen et al. (2006a) could be applied to obtain robust decision rules by taking $(y, q)$ as the observed state. Decision rules that are robust to misspecification of $\varsigma\left(s^{*} \mid y, q, a\right)$ can be obtained by iterating on

$$
W(y, q)=\max _{a \in A} U(y, q, a)+\mathrm{T}^{1}\left[\beta W^{*}\left(y^{*}, q^{*}\right) \mid \theta\right](y, q, a) .
$$

This approach absorbs Bayes' law into the transition law for the state and seeks robustness to misspecification of $\varsigma\left(s^{*} \mid y, q, a\right)$. In contrast, the formulations in (20)-(21) and (23) distinguish distortions to $\kappa\left(s^{*} \mid y, z, a\right)$ and to $q(z)$ and seek robustness to misspecifications of each of them separately.

\subsection{Advantages of our specification}

We take the distribution $q_{t}(z)$ as a state variable and explore misspecifications of it. An alternative way to describe a decision maker's fears of misspecification would be to perturb the evolution equation for the hidden state (2) directly. Doing that would complicate the problem substantially by requiring us to solve a filtering problem for each perturbation of (2). Our formulation avoids multiple filtering problems by solving one and only one filtering problem under the approximating model. The transition law $\pi_{q}$ for $q(z)$ in $(5)$ becomes a component of the approximating model.

When $\theta_{1}=+\infty$ but $\theta_{2}<+\infty$, the decision maker trusts the signal dynamics $\kappa\left(s^{*} \mid y, z, a\right)$ but distrusts $q(z)$. When $\theta_{2}=+\infty$ but $\theta_{1}<+\infty$, the situation is reversed. The two- $\theta$ formulation thus allows the decision maker to disentangle his suspicions about these two aspects of the model. Before saying more about the two- $\theta$ formulation, the next section explores some ramifications of the special case in which $\theta_{1}=\theta_{2}$ and how it compares to the single $\theta$ specification that prevails in a related decision problem under commitment.

\section{The $\theta_{1}=\theta_{2}$ case}

For the purpose of studying intertemporal consistency and other features of the associated worst case models, it is interesting to compare the outcomes of recursions (20)-(21) or (23) with the decision rule and worst case model described by Hansen and Sargent (2005) in which at time 0 the maximizing and minimizing players in a zero-sum game commit to a sequence of decision rules and a single worst case model, respectively. Because there is a single robustness parameter $\theta$ in this "commitment model", it is natural to make this comparison for the special case in which $\theta_{1}=\theta_{2}$. 


\subsection{A composite operator $\mathrm{T}^{2} \circ \mathrm{T}^{1}$ when $\theta_{1}=\theta_{2}$}

When a common value of $\theta$ appears in the two operators, the sequential application $\mathrm{T}^{2} \mathrm{~T}^{1}$ can be replaced by a single operator:

$$
\begin{aligned}
\mathrm{T}^{2} \circ \mathrm{T}^{1}[U(x, a) & \left.+\beta W\left(y^{*}, q^{*}\right)\right](y, q, a) \\
& =-\theta \log \int \exp \left(-\frac{U(x, a)+\beta W\left[\pi\left(s^{*}, y, q, a\right)\right]}{\theta}\right) \kappa\left(s^{*} \mid y, z, a\right) q(z) d \eta\left(s^{*}\right) d \lambda(z) .
\end{aligned}
$$

This operator is the outcome of a portmanteau minimization problem over a single relative density $\varphi\left(s^{*}, z\right) \geq 0$ that satisfies $^{12}$

$$
\int \varphi\left(s^{*}, z\right) \kappa\left(s^{*} \mid y, z, a\right) q(z) d \eta\left(s^{*}\right) d \lambda(z)=1,
$$

where $\varphi$ is related to $\phi$ and $\psi$ defined in (12) and (15) by

$$
\varphi\left(s^{*}, z\right)=\int \phi\left(z^{*}, s^{*} \mid z\right) \psi(z) q^{*}\left(z^{*}\right) d \lambda\left(z^{*}\right)
$$

where this notation emphasizes that the choice of $\phi$ can depend on $z$. The entropy measure for $\varphi$ is

$$
\int\left[\log \varphi\left(s^{*}, z\right)\right] \varphi\left(s^{*}, z\right) \kappa\left(s^{*} \mid y, z, a\right) q(z) d \eta\left(s^{*}\right) d \lambda(z),
$$

and the minimizing composite distortion $\varphi$ to the joint density of $\left(s^{*}, z\right)$ given $\mathcal{S}_{t}$ is

$$
\varphi_{t}\left(s^{*}, z\right)=\frac{\exp \left(-\frac{U\left(y_{t}, z, a_{t}\right)+\beta W\left[\pi\left(s^{*}, y_{t}, q_{t}, a_{t}\right)\right]}{\theta}\right)}{E\left[\exp \left(-\frac{U\left(y_{t}, z, a_{t}\right)+\beta W\left[\pi\left(s_{t+1}, y_{t}, q_{t}, a_{t}\right)\right]}{\theta}\right) \mid \mathcal{S}_{t}\right]} .
$$

\subsection{Special case $U(x, a)=\hat{U}(y, a)$}

When $U(x, a)=\hat{U}(y, a)$, the current period utility drops out of formula (24) for the worstcase distortion to the distribution, and it suffices to integrate with respect to the distribution $\varsigma\left(s^{*} \mid y, q, a\right)$ that we constructed in (4) by averaging $\kappa$ over the distribution of the hidden state. Probabilities of future signals compounded by the hidden state are simply averaged out using the state density under the benchmark model, a reduction of a compound lottery that would not be possible if different values of $\theta$ were to occur in the two operators.

To understand these claims, we deduce a useful representation of $\varepsilon_{t}\left(m_{t+1}, h_{t}\right)$ by solving:

$$
\varepsilon_{t}\left(m_{t+1}^{*}, h_{t}^{*}\right) \equiv \min _{m_{t+1} \in \mathcal{M}_{t}, h_{t} \in \mathcal{H}_{t}} E\left[h_{t} \varepsilon_{t}^{1}\left(m_{t+1}\right) \mid \mathcal{S}_{t}\right]+\varepsilon_{t}^{2}\left(h_{t}\right)
$$

subject to $E\left(m_{t+1} h_{t} \mid \mathcal{S}_{t+1}\right)=g_{t+1}$, where $E\left(g_{t+1} \mid \mathcal{S}_{t}\right)=1$, a constraint that we impose because our aim is to distort expectations of $\mathcal{S}_{t+1}$-measurable random variables given current information $\mathcal{S}_{t}$. The minimizers are

$$
m_{t+1}^{*}=\left\{\begin{array}{cll}
\frac{g_{t+1}}{E\left(g_{t+1} \mid \mathcal{X}_{t}\right)} & \text { if } & E\left(g_{t+1} \mid \mathcal{X}_{t}\right)>0 \\
1 & \text { if } & E\left(g_{t+1} \mid \mathcal{X}_{t}\right)=0
\end{array}\right.
$$

\footnotetext{
${ }^{12}$ Recall that applying $\mathrm{T}^{1}$ and $\mathrm{T}^{2}$ separately amounts to minimizing over separate relative densities $\phi$ and $\psi$.
} 
and $h_{t}^{*}=E\left(g_{t+1} \mid \mathcal{X}_{t}\right)$. Therefore, $m_{t+1}^{*} h_{t}^{*}=g_{t+1}$ and the minimized value of the objective is

$$
\varepsilon_{t}\left(m_{t+1}^{*}, h_{t}^{*}\right)=E\left[g_{t+1} \log \left(g_{t+1}\right) \mid \mathcal{S}_{t}\right] \equiv \tilde{\epsilon}_{t}\left(g_{t+1}\right) .
$$

Thus, in penalizing distortions to continuation values that are $\mathcal{S}_{t}$-measurable, it suffices to use the entropy measure $\tilde{\epsilon}_{t}$ defined in (25) and to explore distortions to the conditional probability of $\mathcal{S}_{t+1}$-measurable events given $\mathcal{S}_{t}$. This is precisely what the $g_{t+1}$ random variable accomplishes. The $g_{t+1}$ associated with $\mathrm{T}^{2} \mathrm{~T}^{1}$ in the special case in which $U(x, a)=$ $\hat{U}(y, a)$ implies a distortion $\phi_{t}$ in equation (13) that depends on $s^{*}$ alone. The iterated operator $\mathrm{T}^{2} \mathrm{~T}^{1}$ can be regarded as a single risk-sensitivity operator that functions like $\mathrm{T}^{1}$ :

$$
\begin{aligned}
\mathrm{T}^{2} \mathrm{~T}^{1}[\hat{U}(y, a) & \left.+\beta W^{*}\left(y^{*}, q^{*}\right)\right](y, q, a) \\
& =\hat{U}(y, a)-\theta \log \int \exp \left(-\frac{\beta W^{*}\left(\pi\left(s^{*}, y, q, a\right)\right)}{\theta}\right) \varsigma\left(s^{*} \mid y, q, a\right) d \eta\left(s^{*}\right) .
\end{aligned}
$$

In Hansen and Sargent (2006b), we describe how to compute this operator for linear quadratic problems.

\subsection{Role of absolute continuity and relation to commitment solu- tions and}

Among the outcomes of iterations on the recursions (20)-(21) or (23) of section 5 are timeinvariant functions that map $\left(y_{t}, q_{t}\right)$ into a pair of nonnegative random variables $\left(m_{t+1}, h_{t}\right)$. For the moment, ignore the distortion $h_{t}$ and focus exclusively on $m_{t+1}$. Through (8), the time-invariant rule for $m_{t+1}$ can be used to a construct a martingale $\left\{M_{t}: t \geq 0\right\}$. This martingale implies a limiting probability measure on $\mathcal{X}_{\infty}=\vee_{t \geq 0} \mathcal{X}_{t}$ via the Kolmogorov extension theorem. The implied probability measure on $\mathcal{X}_{\infty}$ will typically not be absolutely continuous over the entire collection of limiting events in $\mathcal{X}_{\infty}$. Although the martingale converges almost surely by virtue of Doob's martingale convergence theorem, without absolute continuity, the limiting random variable will not have unit expectation. This implies that concerns about robustness persist in a way that they don't in a class of robust control problems under commitment that are studied, for example, by Whittle (1990) and Hansen and Sargent (2005). ${ }^{13}$

\subsubsection{Problem formulation}

Let $M_{\infty}$ be a nonnegative random variable that is measurable with respect to $\mathcal{X}_{\infty}$, with $E\left(M_{\infty} \mid \mathcal{S}_{0}\right)=1$. For a given action process $\left\{a_{t}: t \geq 0\right\}$ adapted to $\left\{\mathcal{X}_{t}: t \geq 0\right\}$, let $V_{\infty} \doteq$

${ }^{13}$ The product decomposition (8) of $M_{t}$ implies an additive decomposition of entropy:

$$
E\left(M_{t} \log M_{t} \mid \mathcal{S}_{0}\right)-E\left(M_{0} \log M_{0} \mid \mathcal{S}_{0}\right)=\sum_{j=0}^{t-1} E\left[M_{j} E\left(m_{j+1} \log m_{j+1} \mid \mathcal{X}_{j}\right) \mid \mathcal{S}_{0}\right] .
$$

Setting $E\left(M_{0} \mid \mathcal{S}_{0}\right)=1$ means that we distort probabilities conditioned on $\mathcal{S}_{0}$. 
$\sum_{t=0}^{\infty} \beta^{t} U\left(x_{t}, a_{t}\right)$ subject to (1)-(2). Suppose that $\theta>0$ is such that $E\left[\exp \left(-\frac{1}{\theta} V_{\infty}\right) \mid \mathcal{S}_{0}\right]<$ $\infty$. Then

$$
\begin{aligned}
\mathrm{R}_{\infty}^{1}\left(V_{\infty}\right) & \doteq \min _{M_{\infty} \geq 0, E\left(M_{\infty} \mid \mathcal{S}_{0}\right)=1} E\left(M_{\infty} V_{\infty} \mid \mathcal{S}_{0}\right)+\theta E\left(M_{\infty} \log M_{\infty} \mid \mathcal{S}_{0}\right) \\
& =-\theta \log E\left[\exp \left(-\frac{1}{\theta} V_{\infty}\right) \mid \mathcal{S}_{0}\right]
\end{aligned}
$$

This static problem has minimizer $M_{\infty}^{*}=\frac{\exp \left(-\frac{1}{\theta} V_{\infty}\right)}{E\left[\exp \left(-\frac{1}{\theta} V_{\infty}\right) \mid \mathcal{S}_{0}\right]}$ that implies a martingale $M_{t}^{*}=$ $E\left(M_{\infty}^{*} \mid \mathcal{X}_{t}\right) .{ }^{14}$ Control theory interprets (28) as a risk-sensitive adjustment of the criterion $V_{\infty}$ (e.g., see Whittle (1990)) and gets decisions that are robust to misspecifications by solving

$$
\max _{a_{t} \in \mathcal{A}_{t}, t \geq 0}-\theta \log E\left[\exp \left(-\frac{1}{\theta} V_{\infty}\right) \mid \mathcal{S}_{0}\right] .
$$

In a closely related setting, Whittle (1990) obtained time-varying decision rules for $a_{t}$ that converge to ones that ignore concerns about robustness (i.e., those computed with $\theta=+\infty$ ).

The dissipation of concerns about robustness in this commitment problem is attributable to setting $\beta \in(0,1)$ while using the undiscounted form of entropy in the criterion function (27). Those features lead to the existence of a well defined limiting random variable $M_{\infty}$ with expectation unity (conditioned on $\mathcal{S}_{0}$ ), which means that tail events that are assigned probability zero under the approximating model are also assigned probability zero under the distorted model. ${ }^{15}$

\subsubsection{Persistence of robustness concerns without commitment}

In our recursive formulations (20)-(21) and (23) of section 5, the failure of the worst-case nonnegative martingale $\left\{M_{t}: t \geq 0\right\}$ to converge to a limit with expectation one (conditioned on $\mathcal{S}_{0}$ ) implies that the distorted probability distribution on $\mathcal{X}_{\infty}$ is not absolutely continuous with respect to the probability distribution associated with the approximating model. This feature sustains enduring concerns about robustness and permits time-invariant robust

\footnotetext{
${ }^{14}$ See Dupuis and Ellis (1997). While robust control problems are often formulated as deterministic problems, here we follow Petersen et al. (2000) by studying a stochastic version with a relative entropy penalty.

${ }^{15}$ Because all terms on the right side of (26) are nonnegative, the sequence

$$
\sum_{j=0}^{t-1} M_{j-1} E\left(m_{j} \log m_{j} \mid \mathcal{X}_{j-1}\right)
$$

is increasing. Therefore, it has a limit that might be $+\infty$ with positive probability. Thus, $\lim _{t \rightarrow \infty} E\left(M_{t} \log M_{t} \mid \mathcal{S}_{0}\right)$ converges. Hansen and Sargent (2005) show that when this limit is finite almost surely, the martingale sequence $\left\{M_{t}: t \geq 0\right\}$ converges in the sense that $\lim _{t \rightarrow \infty} E\left(\left|M_{t}-M_{\infty}\right| \mid \mathcal{S}_{0}\right)=0$, where $M_{\infty}$ is measurable with respect to $\mathcal{X}_{\infty} \doteq \bigvee_{t=0}^{\infty} \mathcal{X}_{t}$. The limiting random variable $M_{\infty}$ can be used to construct a probability measure on $\mathcal{X}_{\infty}$ that is absolutely continuous with respect to the probability measure associated with the approximating model. Moreover, $M_{t}=E\left(M_{\infty} \mid \mathcal{X}_{t}\right)$. When the implied $M_{\infty}$ is strictly positive with probability one, the distorted probability measure will be equivalent with the original probability measure. In this case, tail events that are assigned probability measure zero under either measure are assigned zero under the other one.
} 
decision rules, in contrast to the outcomes with discounting in Whittle (1990) and Hansen and Sargent (2005), for example. For settings with a fully observed state vector, Hansen and Sargent (1995) and Hansen et al. (2006a) discounted entropy in order to formulate recursive problems that yield time-invariant decision rules and enduring concerns about robustness. The present paper extends these recursive formulations to problems with unobserved states.

\subsection{Dynamic inconsistency of worst-case probabilities about hid- den states}

This section links robust control theory to recursive models of uncertainty aversion by exploring aspects of the worst case probability models that emerge from the recursions defined in section 5. Except in a special case that we describe in subsection 6.6, those recursions achieve dynamic consistency of decisions by sacrificing dynamic consistency of beliefs about hidden state variables. We explore how this happens. Until we get to the special case analyzed in subsection 6.6, the arguments of this subsection will also apply to the general case in which $\theta_{1} \neq \theta_{2}$.

Problems (10) and (14) that define $\mathrm{R}_{t}^{1}$ and $\mathrm{R}_{t}^{2}$, respectively, imply worst-case probability distributions that we express as a pair of Radon-Nikodym derivatives $\left(m_{t+1}^{*}, h_{t}^{*}\right)$. Are these probability distortions consistent with next period's distortion $h_{t+1}^{*}$ ? Not necessarily, because we have not imposed the pertinent consistency condition on these beliefs. In particular, our use of $m_{t+1}, h_{t}$ to distort two conditional distributions each period overdetermines a distortion to the distribution of $x_{t+1}$ conditional on $\mathcal{S}_{t+1}$ : because $m_{t+1}$ distorts the probabilities of $\mathcal{X}_{t+1}$ events conditional on $\mathcal{X}_{t}$ and $h_{t}$ distorts the probabilities of $\mathcal{X}_{t}$ events conditioned on $\mathcal{S}_{t}, m_{t+1} h_{t}$ distorts the probabilities of $\mathcal{X}_{t+1}$ events conditional on $\mathcal{S}_{t}$. Given the distorted probabilities of $\mathcal{X}_{t+1}$ events conditioned on $\mathcal{S}_{t}$, we can deduce the probability distortion of $\mathcal{X}_{t+1}$ events conditional on $\mathcal{S}_{t+1}$ (because $\mathcal{S}_{t} \subset \mathcal{S}_{t+1} \subset \mathcal{X}_{t+1}$ ). If we had required the decision maker at time $t+1$ to adhere to this distortion, he would not be free to choose $h_{t+1}$ anew at time $t+1$. Thus, except when a special condition that we lay out in the next subsection is met, the decision maker's worst-case beliefs about the distribution of $x_{t+1}$ conditional on $\mathcal{S}_{t+1}$ will not be time-consistent. This is a price that we pay to attain a recursive formulation in which $q_{t}(z)$ remains a state variable for our formulation of the robust estimation and control problem.

\subsection{A belief consistency condition}

To deduce a sufficient condition for time consistency, recall that the implied $\left\{M_{t+1}^{*}: t \geq 0\right\}$ should be a martingale. Decompose $M_{t+1}^{*}$ in two ways:

$$
M_{t+1}^{*}=m_{t+1}^{*} h_{t}^{*} G_{t}^{*}=h_{t+1}^{*} G_{t+1}^{*} .
$$

These equations involve $G_{t+1}^{*}$ and $G_{t}^{*}$, both of which we have ignored in the recursive formulation of section 5 . Taking expectations conditioned on $\mathcal{S}_{t+1}$ on both sides of $m_{t+1}^{*} h_{t}^{*} G_{t}^{*}=$ $h_{t+1} G_{t+1}^{*}$ yields

$$
G_{t}^{*} E\left(m_{t+1}^{*} h_{t}^{*} \mid \mathcal{S}_{t+1}\right)=G_{t+1}^{*}
$$

Thus,

$$
g_{t+1}^{*}=E\left(m_{t+1}^{*} h_{t}^{*} \mid \mathcal{S}_{t+1}\right)
$$


is the implied multiplicative increment for the candidate martingale $\left\{G_{t}^{*}: t \geq 0\right\}$ adapted to the signal filtration.

Claim 6.1. A sufficient condition for the distorted beliefs to be time consistent is that the process $\left\{h_{t}^{*}: t \geq 0\right\}$ should satisfy:

$$
h_{t+1}^{*}=\left\{\begin{array}{cll}
\frac{m_{t+1}^{*} h_{t}^{*}}{E\left(m_{t+1}^{*} h_{t}^{*} \mathcal{S}_{t+1}\right)} & \text { if } & E\left(m_{t+1}^{*} h_{t}^{*} \mid \mathcal{S}_{t+1}\right)>0 \\
1 & \text { if } & E\left(m_{t+1}^{*} h_{t}^{*} \mid \mathcal{S}_{t+1}\right)=0 .
\end{array}\right.
$$

This condition is necessary if $G_{t+1}^{*}>0 .{ }^{16}$

The robust control problem under commitment analyzed by Hansen and Sargent (2005) satisfies condition (29) by construction: at time 0 a single minimizing player chooses a pair $\left(m_{t+1}^{*}, h_{t}^{*}\right)$ that implies next period's $h_{t+1}^{*}$. However, in the recursive games defined in the recursions (20)-(21) and (23) in section 5, the date $t+1$ minimizing agent can deviate from the $h_{t+1}^{*}$ that is implied by the $\left(m_{t+1}^{*}, h_{t}^{*}\right)$ pair chosen by the date $t$ minimizing agent. The pair $\left(m_{t+1}^{*}, h_{t}^{*}\right)$ gives one distortion of the distribution of the hidden state (conditioned on $\mathcal{S}_{t+1}$ ) and $h_{t+1}^{*}$ gives another. We do not require that these agree, and, in particular, do not require that the probabilities of events in $\mathcal{X}_{t+1}$ be distorted in the same ways by the date $t$ determined worst-case distribution (conditioned on $\mathcal{S}_{t+1}$ ) and the date $t+1$ worst-case distribution (conditioned on $\mathcal{S}_{t+1}$ ).

A conflict can arise between these worst-case distributions because choosing an action is forward-looking, while estimation of $z$ is backward looking. Dynamic inconsistency of any kind is a symptom of conflicts among the interests of different decision makers, and that is the case here. The two-player games that define the evaluation of future prospects $\left(\mathrm{T}^{1}\right)$ and estimation of the current position of the system $\left(\mathrm{T}^{2}\right)$ embody different orientations $-\mathrm{T}^{1}$ looking to the future, $\mathrm{T}^{2}$ focusing on an historical record of signals.

The inconsistency of the worst-case beliefs pertains only to the decision maker's opinions about the hidden state. If we ignore hidden states and focus on signals, we can assemble a consistent distorted signal distribution by constructing $g_{t+1}^{*}=E\left(m_{t+1}^{*} h_{t}^{*} \mid \mathcal{S}_{t+1}\right)$ and noting that $E\left(g_{t+1}^{*} \mid \mathcal{S}_{t}\right)=1$, so that $g_{t+1}^{*}$ is the implied one-period distortion in the signal distribution. We can construct a distorted probability distribution over events in $\mathcal{S}_{t+1}$ by using

$$
G_{t+1}^{*}=\prod_{j=1}^{t+1} g_{j}^{*}
$$

Under this interpretation, the pair $\left(m_{t+1}^{*}, h_{t}^{*}\right)$ is only a device to construct $g_{t+1}^{*}$. When the objective function $U$ does not depend directly on the hidden state vector $z$, as is true in many

\footnotetext{
${ }^{16}$ This consistency condition arguably could be relaxed for the two player game underlying (23). Although we allow $m_{t+1}$ to depend on the signal $s_{t+1}$ and the hidden state $z_{t+1}$, the minimizing solution associated with recursions (23) depends only on the signal $s_{t+1}$. Thus we could instead constrain the minimizing agent in his or her choice of $m_{t+1}$ and introduce a random variable $\tilde{m}_{t+1}$ that distorts the probability distribution of $z_{t+1}$ conditioned on $s_{t+1}$ and $\mathcal{X}_{t}$. A weaker consistency requirement is

$$
h_{t+1}^{*}=\frac{\tilde{m}_{t+1} m_{t+1}^{*} h_{t}^{*}}{E\left(\tilde{m}_{t+1} m_{t+1}^{*} h_{t}^{*} \mid \mathcal{S}_{t+1}\right)}
$$

for some $\tilde{m}_{t+1}$ with expectation equal to one conditioned on $s_{t+1}$ and $\mathcal{X}_{t}$.
} 
economic problems, the consistent set of distorted probabilities defined by (30) describes the events that directly influence the decision maker's well being.

\subsection{Discounting and payoffs influenced by hidden states are the source of intertemporal inconsistency}

If $\beta=1$ and $U(x, a)$ does not depend on the hidden state, we can show that the distortions $\left(m_{t+1}, h_{t}\right)$ implied by our recursions satisfy the restriction required for Claim 6.1 and so are temporally consistent. Therefore, in this special case, the recursive games in section 5 imply the same decisions and worst case distortions as the game under commitment analyzed by Hansen and Sargent (2005). For simplicity, suppose that we fix an action process $\left\{a_{t}: t \geq 0\right\}$ and focus exclusively on assigning distorted probabilities. Let $\left\{V_{t}: t \geq 0\right\}$ denote the process of continuation values determined recursively and supported by choices of worst-case models.

Consider two operators $\mathrm{R}_{t}^{1}$ and $\mathrm{R}_{t}^{2}$ with a common $\theta$. The operator $\mathrm{R}_{t}^{1}$ implies a worst-case distribution for $\mathcal{X}_{t+1}$ conditioned on $\mathcal{X}_{t}$ with density distortion:

$$
m_{t+1}^{*}=\frac{\exp \left(-\frac{V_{t+1}}{\theta}\right)}{E\left[\exp \left(-\frac{V_{t+1}}{\theta}\right) \mid \mathcal{X}_{t}\right]} .
$$

The operator $\mathrm{R}_{t}^{2}$ implies a worst-case model for the probability of $\mathcal{X}_{t}$ conditioned on $\mathcal{S}_{t}$ with density distortion:

$$
h_{t}^{*}=\frac{E\left[\exp \left(-\frac{V_{t+1}}{\theta}\right) \mid \mathcal{X}_{t}\right]}{E\left[\exp \left(-\frac{V_{t+1}}{\theta}\right) \mid \mathcal{S}_{t}\right]} .
$$

Combining the distortions gives

$$
m_{t+1}^{*} h_{t}^{*}=\frac{\exp \left(-\frac{V_{t+1}}{\theta}\right)}{E\left[\exp \left(-\frac{V_{t+1}}{\theta}\right) \mid \mathcal{S}_{t}\right]} .
$$

To establish temporal consistency, from Claim 6.1 we must show that

$$
h_{t+1}^{*}=\frac{\exp \left(-\frac{V_{t+1}}{\theta}\right)}{E\left[\exp \left(-\frac{V_{t+1}}{\theta}\right) \mid \mathcal{S}_{t+1}\right]}
$$

where

$$
h_{t+1}^{*} \doteq \frac{E\left[\exp \left(-\frac{V_{t+2}}{\theta}\right) \mid \mathcal{X}_{t+1}\right]}{E\left[\exp \left(-\frac{V_{t+2}}{\theta}\right) \mid \mathcal{S}_{t+1}\right]} .
$$

This relation is true when $\beta=1$ and $U$ does not depend on the hidden state $z$. To accommodate $\beta=1$, we shift from an infinite horizon problem to a finite horizon problem with a terminal value function. From value recursion (20) and the representation of $\mathrm{R}_{t+1}^{1}$ in (10),

$$
\exp \left(-\frac{V_{t+1}}{\theta}\right) \propto E\left[\exp \left(-\frac{V_{t+2}}{\theta}\right) \mid \mathcal{X}_{t+1}\right]
$$


where the proportionality factor is $\mathcal{S}_{t+1}$ measurable. The consistency requirement for $h_{t+1}^{*}$ is therefore satisfied.

The preceding argument isolates the role that discounting plays in rendering the worst case beliefs over the hidden state time inconsistent. Heuristically, the games defined by the recursions (20)-(21) or (23) imply intertemporal inconsistency when $\beta<1$ because the decision maker discounts both current period returns and current period increments to entropy; while in the commitment problem analyzed in Hansen and Sargent (2005), the decision maker discounts current period returns but not current period increments to entropy.

\section{Implied worst case model of signal distortion}

The martingale (relative to $\left.\mathcal{S}_{t}\right)$ increment $g_{t+1}=E\left(m_{t+1} h_{t} \mid \mathcal{S}_{t}\right)$ distorts the distribution of the date $t+1$ signal given information $\mathcal{S}_{t}$ generated by current and past signals. For the following three reasons, it is interesting to construct an implied $g_{t+1}^{*}$ from the $m_{t+1}^{*}$ associated with $\mathrm{R}_{t}^{1}$ or $\mathrm{T}^{1}$ and the $h_{t}^{*}$ associated with $\mathrm{R}_{t}^{2}$ or $\mathrm{T}^{2}$.

First, actions depend only on signal histories. Hidden states are used either to depict the underlying uncertainty or to help represent preferences. However, agents cannot take actions contingent on these hidden states, only on the signal histories.

Second, in decentralized economies, asset prices can be characterized by stochastic discount factors that equal the intertemporal marginal rates of substitution of investors who are off corners and that depend on the distorted probabilities these investors use to value contingent claims. Since contingent claims to consumption can depend only on signal histories (and not on hidden states), the distortion to the signal distribution is the twist to asset pricing that is contributed by investors' concerns about model misspecification. In particular, under the approximating model, $\frac{g_{t+1}}{E\left[g_{t+1} \mid \mathcal{S}_{t}\right]}$ becomes a multiplicative adjustment to the ordinary stochastic discount factor for a representative agent (e.g., see Hansen et al. (1999)) It follows that the temporal inconsistency of worst case beliefs over hidden states discussed in section 6.4 does not prevent appealing to standard results on the recursive structure of asset pricing in settings with complete markets. ${ }^{17}$

Third, Anderson et al. (2003) found it useful to characterize detection probabilities using relative entropy and an alternative measure of entropy due to Chernoff (1952). Chernoff (1952) showed how detection error probabilities for competing models give a way to measure model discrepancy. Models are close when they are hard to distinguish with historical data. Because signal histories contain all data that are available to a decision maker, the measured entropy from distorting the signal distribution is pertinent for statistical discrimination. These lead us to measure either $E\left(g_{t+1}^{*} \log g_{t+1}^{*} \mid \mathcal{S}_{t}\right)$ or Chernoff's counterpart, as in Anderson et al. (2003). ${ }^{18}$

Our characterizations of worst case models have conditioned implicitly on the current period action. The implied distortion in the signal density is:

$$
\int \phi_{t}\left(z^{*}, s^{*}\right) \tau\left(z^{*}, s^{*} \mid y_{t}, z,, a_{t}\right) \psi_{t}(z) q_{t}(z) d \lambda\left(z^{*}\right) d \lambda(z)
$$

\footnotetext{
${ }^{17}$ See Johnsen and Donaldson (1985).

${ }^{18}$ Anderson et al. (2003) show a close connection between the market price of risk and a bound on the error probability for a statistical test for discriminating the approximating model from the worst case model.
} 
where $\phi_{t}$ is given by formula (13) and $\psi_{t}$ is given by (16). When a Bellman-Isaacs condition is satisfied, ${ }^{19}$ we can substitute for the control law and construct a conditional worst case conditional probability density for $s_{t+1}$ as a function of the Markov state $\left(y_{t}, q_{t}\right)$. The process $\left\{\left(y_{t+1}, q_{t+1}\right): t \geq 0\right\}$ is Markov under the worst case distribution for the signal evolution. The density $q_{t}$ remains a component of the state vector.

\section{A recursive multiple priors model}

To attain a notion of dynamic consistency when the decision maker has multiple models, Epstein and Schneider (2003a,b) advocate a formulation that, when translated into our setting, implies time varying values for $\theta_{1}$ and $\theta_{2}$. Epstein and Schneider advocate sequential constraints on sets of transition probabilities for signal distributions. To implement their proposal in our context, we can replace our fixed penalty parameters $\theta_{1}, \theta_{2}$ with two sequences of constraints on relative entropy.

In particular, suppose that

$$
\varepsilon_{t}^{1}\left(m_{t+1}\right) \leq \kappa_{t}^{1}
$$

where $\kappa_{t}^{1}$ is a positive random variable in $\mathcal{X}_{t}$, and

$$
\varepsilon_{t}^{2}\left(h_{t}\right) \leq \kappa_{t}^{2}
$$

where $\kappa_{t}^{2}$ is a positive random variable in $\mathcal{S}_{t}$. If these constraints bind, the worst-case probability distributions are again exponentially tilted. We can take $\theta_{t}^{1}$ to be the $\mathcal{X}_{t^{-}}$ measurable Lagrange Multiplier on constraint (31), where $m_{t+1}^{*} \propto \exp \left(-\frac{W_{t+1}}{\theta_{t}^{1}}\right)$ and $\theta_{t}^{1}$ solves $\varepsilon_{t}^{1}\left(m_{t+1}^{*}\right)=\kappa_{t}^{1}$. The counterpart to $\mathrm{R}_{\mathrm{t}}^{1}\left(\mathrm{~W}_{\mathrm{t}+1}\right)$ is

$$
\mathrm{C}_{\mathrm{t}}^{1}\left(\mathrm{~W}_{\mathrm{t}+1}\right) \doteq \frac{\mathrm{E}\left[\mathrm{W}_{\mathrm{t}+1} \exp \left(-\frac{\mathrm{W}_{\mathrm{t}+1}}{\theta_{\mathrm{t}}^{1}}\right) \mid \mathcal{X}_{\mathrm{t}}\right]}{\mathrm{E}\left[\exp \left(-\frac{\mathrm{W}_{\mathrm{t}+1}}{\theta_{\mathrm{t}}^{1}}\right) \mid \mathcal{X}_{\mathrm{t}}\right]}
$$

Similarly, let $\theta_{t}^{2}$ be the $\mathcal{S}_{t}$-measurable Lagrange multiplier on constraint (32), where $h_{t}^{*} \propto$ $\exp \left(-\frac{\hat{W}_{t}}{\theta_{t}^{2}}\right)$, and $\theta_{t}^{2}$ solves $\varepsilon_{t}^{2}\left(h_{t}^{*}\right)=\kappa_{t}^{2}$. The counterpart to $\mathrm{R}_{\mathrm{t}}^{2}\left(\hat{\mathrm{W}}_{\mathrm{t}}\right)$ is

$$
\mathrm{C}_{\mathrm{t}}^{2}\left(\hat{\mathrm{W}}_{\mathrm{t}}\right) \doteq \frac{\mathrm{E}\left[\hat{\mathrm{W}}_{\mathrm{t}} \exp \left(-\frac{\hat{\mathrm{W}}_{\mathrm{t}}}{\theta_{\mathrm{t}}^{2}}\right) \mid \mathcal{S}_{\mathrm{t}}\right]}{\mathrm{E}\left[\exp \left(-\frac{\hat{\mathrm{W}}_{\mathrm{t}}}{\theta_{\mathrm{t}}^{2}}\right) \mid \mathcal{S}_{\mathrm{t}}\right]}
$$

These constraint problems lead to natural counterparts to the operators $\mathrm{T}^{1}$ and $\mathrm{T}^{2}$.

Constraint formulations provide a justification for making $\theta_{1}$ and $\theta_{2}$ state- or timedependent. Values of $\theta_{1}$ and $\theta_{2}$ would coincide if the two constraints were replaced by a single entropy constraint $E\left[h_{t} \varepsilon_{t}^{1}\left(m_{t+1}\right) \mid \mathcal{S}_{t}\right]+\varepsilon_{t}^{2}\left(h_{t}\right) \leq \kappa_{t}$, where $\kappa_{t}$ is $\mathcal{S}_{t}$-measurable. Lin et al. (2004) and Maenhout (2004) give other reasons for making the robustness penalty parameters state dependent. ${ }^{20}$ With such state dependence, it can still be useful to disentangle

\footnotetext{
${ }^{19}$ For example, see Hansen et al. (2006a) or Hansen and Sargent (2006d).

${ }^{20}$ These authors consider problems without hidden states, but their motivation for state dependence would carry over to decision problems with hidden states.
} 
misspecifications of the state dynamics and the distribution of the hidden state given current information. Using separate values for $\theta_{1}$ and $\theta_{2}$ achieves that.

\section{$9 \quad$ Risk sensitivity and compound lotteries}

Jacobson (1973) linked a concern about robustness, as represented in the first line of (10), to risk sensitivity, as conveyed in the second line of (10). That link has been exploited in the control theory literature, for example, by Whittle (1990). Our desire to separate the concern about misspecified state dynamics from concern about misspecifying the distribution of the state inspires two risk-sensitivity operators. Although our primary interest is to let a decision maker respond to model misspecification, our two operators can also be interpreted in terms of enhanced risk aversion. ${ }^{21}$

\subsection{Risk-sensitive interpretation of $\mathrm{R}_{t}^{1}$}

The $\mathrm{R}_{t}^{1}$ operator has an alternative interpretation as a risk-sensitive adjustment to continuation values that expresses how a decision maker who has no concern about robustness prefers to adjust continuation values for their risk. The literature on risk-sensitive control uses adjustments of the same $\log E \exp$ form that emerge from an entropy penalty and a concern for robustness, as asserted in (10). There are risk adjustments that are more general than those of the $\log E \exp$ form associated with risk-sensitivity. In particular, we could follow Kreps and Porteus (1978) and Epstein and Zin (1989) in relaxing the assumption that a temporal compound lottery can be reduced to a simple lottery without regard to how the uncertainty is resolved, which would lead us to adjust continuation values by

$$
\tilde{\mathrm{R}}_{t}^{1}\left(V_{t+1}\right)=\phi^{-1}\left(E\left[\phi\left(V_{t+1}\right) \mid \mathcal{X}_{t}\right]\right)
$$

for some concave increasing function $\phi$. The risk-sensitive case is the special one in which $\phi$ is an exponential function. We focus on the special risk-sensitivity $\log E \exp$ adjustment because it allows us to use entropy to interpret the resulting adjustment as a way of inducing robust decision rules.

\section{2 $\mathrm{R}_{t}^{2}$ and the reduction of compound lotteries}

While (16) shows that the operator $\mathrm{R}_{t}^{2}$ assigns a worst-case probability distribution, another interpretation along the lines of Segal (1990), Klibanoff et al. (2003), and Ergin and Gul (2004) is available. This operator adjusts for state risk differently than does the usual Bayesian model averaging approach. Specifically, we can regard the transformation $\mathrm{R}_{t}^{2}$ as a version of what Klibanoff et al. (2003) call constant ambiguity aversion. More generally, we could use

$$
\tilde{\mathrm{R}}_{t}^{2}\left(\hat{V}_{t}\right)=\psi^{-1} E\left[\psi\left(\hat{V}_{t}\right) \mid \mathcal{S}_{t}\right]
$$

for some concave increasing function $\psi$. Again, we use the particular 'log $E$ exp' adjustment because of its explicit link to entropy-based robustness.

\footnotetext{
${ }^{21}$ Using detection probabilities, Anderson et al. (2003) describe alternative senses in which the risksensitivity and robustness interpretations are and are not observationally equivalent.
} 


\section{Another example}

Hansen and Sargent (2006a) follow Tallarini (2000) and start with a representative consumer who, if he did not want to make a risk-sensitivity or robustness adjustment, would value streams of $\log$ consumption $c_{t}$ according to

$$
V_{t}=(1-\beta) c_{t}+E_{t} \beta V_{t+1}
$$

But he wants to make multiple risk sensitivity adjustments to reflect multiple doubts about the stochastic specification of consumption growth. The representative consumer has two submodels for consumption growth $s_{t+1} \equiv c_{t+1}-c_{t}$, each of which has the state space form

$$
\begin{aligned}
\zeta_{t+1}(\iota) & =A(\iota) \zeta_{t}(\iota)+C(\iota) w_{t+1} \\
c_{t+1}-c_{t} \equiv s_{t+1} & =D(\iota) \zeta_{t}(\iota)+G(\iota) w_{t+1}
\end{aligned}
$$

where $\left\{w_{t+1}\right\}$ is an iid Gaussian process with mean 0 and covariance $I$ and $\zeta_{0}(\iota)$ is normally distributed with mean $\zeta_{0}(\iota)$ and covariance matrix $\Sigma_{0}(\iota)$. Denote the submodels $\iota \in\{0,1\}$ and suppose that the representative consumer attaches probability $\check{p}_{t}=E\left(\iota \mid \mathcal{S}_{t}\right)$ to model 1 at time $t$ These probabilities can be computed by using Bayes rule and data $s^{t}=\left[s_{t}, s_{t-1}, \ldots, s_{1}\right]$.

Hansen and Sargent (2006a) specify submodel $\iota=0$ so that it makes consumption growth be an i.i.d. Gaussian process with an unknown mean. Submodel $\iota=1$ is like, but not identical to, a model of Bansal and Yaron (2004) that makes consumption growth contain a difficult to detect persistent component. In addition to the uncertainty about shocks $w_{t+1}$ assumed by Bansal and Yaron, one component of $\zeta(1)$ is a constant conditional mean of consumption that is unknown to the representative consumer. This feature would increase the risk faced by our representative consumer relative to Bansal and Yaron's, even if he set $\hat{p}_{0}=1$. The representative learns about the mean consumption growth parameters as well as other parts of the hidden state $z_{t}=\left[\zeta_{t}(0), \zeta_{t}(1), \iota\right]$.

The results of applying Bayes' law to submodel $\iota$ can be represented in terms of an innovations representation that takes the form

$$
\begin{aligned}
\check{\zeta}_{t+1}(\iota) & =A(\iota) \check{\zeta}_{t}(\iota)+K\left[\Sigma_{t}(\iota), \iota\right] \check{w}_{t+1}(\iota) \\
\Sigma_{t+1}(\iota) & =A(\iota) \Sigma_{t}(\iota) A(\iota)^{\prime}+C(\iota) C(\iota)^{\prime}-K\left[\Sigma_{t}(\iota), \iota\right]\left[A(\iota) \Sigma_{t}(\iota) A(\iota)^{\prime}+C(\iota) G(\iota)^{\prime}\right]^{\prime} \\
s_{t+1} & =D(\iota) \check{\zeta}_{t}+\check{w}_{t+1}(\iota)
\end{aligned}
$$

where

$$
K\left[\Sigma_{t}(\iota), \iota\right] \doteq\left[A(\iota) \Sigma_{t}(\iota) D(\iota)^{\prime}+C(\iota) G(\iota)^{\prime}\right]\left[D(\iota) \Sigma_{t}(\iota) D(\iota)^{\prime}+G(\iota) G(\iota)^{\prime}\right]^{-1}
$$

$\check{\zeta}_{t+1}(\iota)=E\left[\zeta_{t+1} \mid s^{t}, \iota\right], \check{w}_{t+1}(\iota)$ is the forecast error for the signal (i.e., the 'innovation'), and $\Sigma_{t}(\iota)$ is the covariance matrix for $\zeta_{t}(\iota)-\check{\zeta}_{t}(\iota)$ conditioned on $\iota$ and the signal history through date $t$. Evidently, in this model, $\check{\zeta}_{t}(\iota), \Sigma_{t}(\iota), \iota=0,1$, and $\check{p}_{t}$ are sufficient statistics for the joint distribution $q_{t}(z)$.

Hansen and Sargent (2006a) apply recursions (20), (21) to form the stochastic discount factor implied by a representative consumer who is concerned about misspecifications of the 
following distributions: (i) the distributions of $\left(z_{t+1}, s_{t+1}\right)$ conditioned on $\left[\iota, \zeta_{t}(\iota)\right]$; (ii) the distributions of $\zeta_{t}(\iota)$ conditioned on $\left[\iota, \mathcal{S}_{t}\right]$; and (iii) the distributions of $\iota$, conditional on $\mathcal{S}_{t}$. The representative consumer of Hansen and Sargent (2006a) applies $\mathrm{T}^{1}$ to adjust for his suspicion about (i) and iterates on (20) to find valuations as functions of $\zeta(\iota), \iota$. The representative consumer makes adjustment (21) by applying $\mathrm{T}^{2}$ first to adjust the distribution mentioned in (ii). He then applies another $\mathrm{T}^{2}$ operator to adjust for suspicion of the distribution mentioned in (iii). The implied Radon-Nikodym derivative that perturbs the distribution of $s_{t+1}=c_{t+1}-c_{t}$ conditional on $\mathcal{S}_{t}$ serves as a multiplicative adjustment to the stochastic discount factor; in a $T^{1}$-only model, Hansen et al. (1999) dubbed its conditional standard deviation the market price of model uncertainty. Hansen and Sargent (2006a) study market prices of model uncertainty that emerge from the setting described here and investigate how it compares to ones that emerge from the $\mathrm{T}^{1}$ only models of Hansen et al. (1999) and Tallarini (2000).

The distributions mentioned in (i) and (ii) of the previous paragraph are both Gaussian, while the one in (iii) is a scalar $\in(0,1)$. Because the logarithmic preference specification, the value function for problem posed in section 5.1 is affine in $\check{\zeta}, c$. As a result the calculations in this model become very easy - the Kalman filter does the hard work in implementing Bayes' Law and the calculations of $\mathrm{T}^{1}, \mathrm{~T}^{2}$ for the linear-quadratic Gaussian model in Hansen and Sargent (2006c) apply. The assumption that $A(\iota), C(\iota), D(\iota), G(\iota)$ are known accounts for this simplicity. Extending the model to let some elements in these matrices be unknown enriches the scope for modeling learning about unknown parameters at the cost of making the filtering problem nonlinear and so pushing it beyond the range of the Kalman filter. Hansen et al. (2006b) study such problems.

\section{Concluding remarks}

By incorporating learning, this paper responds to thoughtful criticisms of our earlier work about recursive formulations of robust control without learning. In the context of concrete examples, the framework here allows us to examine the consequences for valuations and decision rules of learnable components of the state that can capture both model selection and parameter estimation. For example,

The model in Hansen and Sargent (2006a) that we described in section 10 is about a pure endowment economy, so that the representative consumer chooses no actions - his worst case model determines valuations but not actions. Of course, the framework in this paper allows us also to study settings in which a decision maker chooses an action that influences the motion of the state. We illustrate this aspect by performing an analysis of robust experimentation in Cogley et al. (2005a). For a given concern about misspecification of hidden state probabilities as measured by $\theta_{2}$, we can study the speed at which learning works to diminish concerns about misspecification along particular dimensions of uncertainty as the accretion of data together with Bayes law gradually reduces the set of perturbed models by tightening posterior probabilities. The formulas in Hansen and Sargent (2006b) and Hansen and Sargent (2006a) show precisely how the volatilities of hidden state estimates that come from Bayes' law affect the gap between the worst case probabilities and those from the approximating model. 
Our procedures for solving robust discounted dynamic programming problems are as easy to use as corresponding problems without concerns about robustness and come down to replacing each of two conditional expectations operators in the problem without robustness with a risk-sensitivity operator. For a finite $\theta_{1}$, the operator $\mathrm{T}^{1}$ captures the decision maker's fear that the state and signal dynamics conditioned on both the observed and hidden components of the state are misspecified. For a finite $\theta_{2}$, the operator $\mathrm{T}^{2}$ captures the decision maker's fear that the distribution of the hidden state conditioned on the history of signals is misspecified. Using different values of $\theta_{1}$ and $\theta_{2}$ in the operators $\mathrm{T}^{1}$ and $\mathrm{T}^{2}$ gives us the freedom to focus distrust on different aspects of the decision maker's model. ${ }^{22}$

\footnotetext{
${ }^{22}$ Specifications with $\theta_{1}=\theta_{2}$ emerge when we follow Hansen and Sargent (2005) by adopting a timing protocol that requires the malevolent agent to commit to a worst case model $\left\{M_{t+1}\right\}$ once and for all at time 0 . Hansen and Sargent (2005) give a recursive representation for the solution of the commitment problem in terms of $R_{t}^{1}$ and $R_{t}^{2}$ operators with a common but time-varying multiplier equal to $\frac{\theta}{\beta^{t}}$. The presence of $\beta^{t}$ causes the decision maker's concerns about misspecification to vanish for tail events. Only for the undiscounted case does the zero-sum two player game with commitment in Hansen and Sargent (2005) give identical outcomes to the games without commitment in this paper. As noted in section 6.6 , when $\beta<1$, the gap between the outcomes with and without commitment is the source of time-inconsistency of the worst case beliefs about the hidden state. Much of the control theory literature (e.g., Whittle (1990) and Başar and Bernhard (1995)) uses the commitment timing protocol and sets $\beta=1$. Hansen and Sargent (2005) show how to represent parts of that literature in terms of our formulation of model perturbations as martingales.
} 


\section{References}

Anderson, E., L.P. Hansen, and T.J. Sargent. 2003. A Quartet of Semigroups for Model Specification, Robustness, Prices of Risk, and Model Detection. Journal of the European Economic Association 1 (1):68-123.

Bansal, Ravi and Amir Yaron. 2004. Risks for the Long Run: A Potential Resolution of Asset Pricing Puzzles. Journal of Finance LIX (4):1481-1509.

Başar, T. and P. Bernhard. 1995. $H^{\infty}$-Optimal Control and Related Minimax Design Problems. Birkhauser, second ed.

Bergemann, Dirk and J. Valimaki. 1996. Learning and Strategic Pricing. Econometrica 64:1125-1149.

Brock, William A., Steven N. Durlauf, and Kenneth D. West. 2003. Policy Evaluation in Uncertain Economic Environments.

Brock, William A., Steven Durlauf, and Kenneth D. West. 2004. Model Uncertainty and Policy Evaluation: Some Theory and Empirics. University of Wisconsin, SSRI paper number 2004-19.

Chernoff, Herman. 1952. A Measure of Asymptotic Efficiency for Tests of a Hypothesis Based on the Sum of Observations. Annals of Statistics 23:493-507.

Cogley, Timothy, Riccardo Colacito, Lars Hansen, and Thomas Sargent. 2005a. Robustness and U.S. Monetary Policy Experimentation. Unpublished.

Cogley, Timothy, Riccardo Colacito, and Thomas Sargent. 2005b. Benefits from U.S. Monetary Policy Experimentation in the Days of Samuelson and Solow and Lucas. Unpublished.

Dupuis, P. and R. S. Ellis. 1997. A Weak Convergence Approach to the Theory of Large Deviations. Wiley Series in Probability and Statistics. New York: John Wiley and Sons.

Elliott, R. J., L. Aggoun, and J. B. Moore. 1995. Hidden Markov Models: Estimation and Control. New York: Springer-Verlag.

Epstein, L. and M. Schneider. 2003a. Independently and Indistinguishably Distributed. Journal of Economic-Theory 113 (1):32-50.

—. 2003b. Recursive Multiple Priors. Journal of Economic Theory 113 (1):1-31.

Epstein, L. and S. Zin. 1989. Substitution, Risk Aversion and the Temporal Behavior of Consumption and Asset Returns: A Theoretical Framework. Econometrica 57:937-969.

Ergin, H. and F. Gul. 2004. A Subjective Theory of Compound Lotteries. Unpublished.

Hansen, L. P., T. J. Sargent, G. A. Turmuhambetova, and N. Williams. 2006a. Robust Control, Min-Max Expected Utility, and Model Misspecification. Journal of Economic Theory Forthcoming. 
Hansen, Lars Peter and Thomas Sargent. 1995. Discounted Linear Exponential Quadratic Gaussian Control. IEEE Transactions on Automatic Control 40 (5):968-971.

Hansen, Lars Peter and Thomas J. Sargent. 2005. Robust Estimation and Control under Commitment. Journal of Economic Theory 124 (2):258-301.

- 2006a. Fragile beliefs and the price of model uncertainty. Unpublished.

—. 2006b. Robust Estimation and Control for LQ Gaussian problems without Commitment. Unpublished.

- 2006c. Robust Estimation and Control for LQ Gaussian Problems without Commitment. Unpublished.

—. 2006d. Robustness. Princeton University Press, forthcoming.

Hansen, Lars Peter, Thomas Sargent, and Thomas Tallarini. 1999. Robust Permanent Income and Pricing. Review of Economic Studies 66:873-907.

Hansen, Lars Peter, Nicholas Polson, and Thomas J. Sargent. 2006b. Fragile Beliefs with parameter estimation. Unpublished.

Jacobson, D. H. 1973. Optimal Stochastic Linear Systems with Exponential Performance Criteria and Their Relation to Deterministic Differential Games. IEEE Transactions for Automatic Control AC-18:1124-131.

Johnsen, T. H. and J. B. Donaldson. 1985. The Structure of Intertemporal Preferences under Uncertainty and Time Consistent Plans. Econometrica 53:1451-1458.

Jovanovic, Boyan. 1979. Job Matching and the Theory of Turnover. Journal of Political Economy 87 (5):972-990.

- 1982. Selection and the Evolution of Industry. Econometrica 50 (3):649-670.

Jovanovic, Boyan and Yaw Nyarko. 1995. The Transfer of Human Capital. Journal of Economic Dynamics and Control 19:1033-1064.

- 1996. Learning by Doing and the Choice of Technology. Econometrica 64 (6):12991310.

Klibanoff, P., M. Marinacci, and S. Mukerji. 2003. A Smooth Model of Decision Making Under Ambiguity. Northwestern University.

Kreps, D. M. and E. L. Porteus. 1978. Temporal Resolution of Uncertainty and Dynamic Choice. Econometrica 46:185-200.

Laibson, David. 1997. Golden Eggs and Hyperbolic Discounting. Quarterly Journal of Economics 112 (2):443-478.

Lin, J., J. Pan, and T. Wang. 2004. An Equilibrium Model for Rare-Event Premia and its Implication for Option Pricing. Review of Financial Studies forthcoming. 
Maenhout, P. J. 2004. Robust Portfolio Rules and Asset Pricing. Review of Financial Studies forthcoming.

Petersen, I. R., M. R. James, and P. Dupuis. 2000. Minimax Optimal Control of Stochastic Uncertain Systems with Relative Entropy Constraints. IEEE Transactions on Automatic Control 45:398-412.

Segal, U. 1990. Two-Stage Lotteries without the Reduction Axiom. Econometrica 58:349377.

Tallarini, Thomas D. 2000. Risk-sensitive real business cycles. Journal of Monetary Economics 45 (3):507-532.

Weiland, Volker. 2005. Comment on 'Certainty Equivalence and Model Uncertainty'. In Models and Monetary Policy: Research in the Tradition of Dale Henderson, Richard Porter and Peter Tinsley, edited by Athanasios Orphanides, David Reifschneider, and Jonathan Faust. Washington, D.C.: Board of Governors of the Federal Reserve System.

Whittle, Peter. 1990. Risk-Sensitive Optimal Control. New York: John Wiley \& Sons.

Wonham, W. J. 1964. Some Applications of Stochastic Differential Equations to Optimal Nonlinear Filtering. Siam Journal of Control 2 (3):347-368. 